\title{
Suppressed expression of miR-378 targeting gzmb in NK cells is required to control dengue virus infection
}

\author{
Shuyan Liu ${ }^{1}$, Lingming Chen ${ }^{1}$, Ying Zeng ${ }^{2}$, Lulu Si ${ }^{1}$, Xiaolan Guo ${ }^{1}$, Junmei Zhou ${ }^{1}$, Danyun Fang ${ }^{1}$, \\ Gucheng Zeng ${ }^{1}$ and Lifang Jiang ${ }^{1}$
}

Dengue virus (DENV) remains a major public health threat because no vaccine or drugs are available for the prevention and treatment of DENV infection, and the immunopathogenesis mechanisms of DENV infection are not fully understood. Cytotoxic molecules, such as granzyme B (GrzB), may be necessary to control viral infections. However, the exact role of GrzB during DENV infection and the mechanisms regulating GrzB expression during DENV infection are not clear. This study found that miR-27a*, miR-30e, and miR-378 were down-regulated in DENV-infected patients, and DENV infection in humans induced a significant up-regulation of GrzB in natural killer (NK) cells and CD8 ${ }^{+} \mathrm{T}$ cells. Further investigation indicated that NK cells, but not $\mathrm{CD8}^{+} \mathrm{T}$ cells, were the major sources of GrzB, and miR-378, but not miR-27a* or miR-30e, suppressed GrzB expression in NK cells. Notably, we found that overexpression of miR-378 using a miR-378 agomir in DENV-infected mice inhibited GrzB expression and promoted DENV replication. These results suggest the critical importance of miR-378 in the regulation of GrzB expression and a protective role for GrzB in controlling DENV replication in vivo. Therefore, this study provides a new insight into the immunopathogenesis mechanism of DENV infection and a biological basis for the development of new therapeutic strategies to control DENV infection.

Cellular \& Molecular Immunology advance online publication, 13 July 2015; doi:10.1038/cmi.2015.52

Keywords: dengue virus; granzyme B; miRNA-378; NK cells

\section{INTRODUCTION}

Dengue virus (DENV) is a positive-polarity, single-stranded RNA virus in the mosquito-borne flavivirus family. DENV has four infectious serotypes: DENV-1, $-2,-3$, and -4 . DENV infection results in clinical diseases ranging from an asymptomatic, acute self-limiting febrile illness, such as dengue fever, to a much more severe life-threatening form of dengue infection that is characterized by high fever, large-scale hemorrhage, plasma leakage, and multiple organ failure, such as dengue hemorrhagic fever (DHF)/dengue shock syndrome (DSS). ${ }^{1,2}$ More than 50 million people annually contract DENV, which leads to approximately 500000 hospitalizations and 25000 deaths, particularly children. ${ }^{3}$ Therefore, DENV remains a serious public health threat in tropical and subtropical areas. ${ }^{3}$ Unfortunately, there are no drugs or vaccines that target DENV. Elucidation of the immunopathogenesis mechanisms of DENV infection is critically important for the development of anti-DENV drugs and vaccines.
DENV infection induces massive immune activation and the production of high amounts of proinflammatory cytokines, such as tumor necrosis factor-alpha (TNF- $\alpha$ ) and interferongamma (IFN- $\gamma$ ), which may contribute to the immunopathogenesis of severe DENV infection, such as DHF/DSS. ${ }^{4-6}$ Cellular cytotoxic molecules, such as perforin and granzymes, may also contribute to the progression of DENV infection and the development of massive vascular leaks that lead to DHF/ DSS, ${ }^{7,8}$ particularly in secondary dengue infection with heterologous serotypes.

Natural killer (NK) cells, $\mathrm{CD} 8^{+} \mathrm{T}$ cells, and a few $\mathrm{CD} 4^{+} \mathrm{T}$ cells are the major sources of perforin and GrzB expression. ${ }^{9,10}$ NK cells and DENV-specific $\mathrm{CD} 8^{+} \mathrm{T}$ cells are likely activated at a very early stage during an acute DENV infection. ${ }^{11}$ These cells produce cytokines, cytotoxic molecules, and adhesion molecules ${ }^{11-14}$ and demonstrate potent cytotoxic activity, ${ }^{14}$ which may promote the development of an efficient adaptive immune response by $\mathrm{CD}^{+}{ }^{+} \mathrm{T}$ cells. ${ }^{15} \mathrm{NK}$ cells and DENV-specific CD8 ${ }^{+}$

\footnotetext{
${ }^{1}$ Department of Microbiology, Zhongshan School of Medicine, Key Laboratory for Tropical Diseases Control of the Ministry of Education, Sun Yat-sen University, Guangzhou 510080, China and ²Department of Neurology, The First Affiliated Hospital, Sun Yat-sen University, Guangzhou 510080, China Correspondence: G Zeng and L Jiang, Department of Microbiology, Zhongshan School of Medicine, Key Laboratory for Tropical Diseases Control of the Ministry of Education, Sun Yat-sen University, Guangzhou 510080, China E-mail: zenggch@mail.sysu.edu.cn (to G.Z.) or jianglf@mail.sysu.edu.cn (to L.J.).
}

Received: 9 March 2015; Revised: 14 May 2015; Accepted: 15 May 2015 
$\mathrm{T}$ cells participate in either the control of viral replication or the promotion of the immunopathogenesis that leads to DHF/DSS. ${ }^{11,12,14}$ However, use of the perforin/GrzB pathway by NK cells and $\mathrm{CD} 8^{+} \mathrm{T}$ cells to control DENV infection or promote the immunopathogenesis that leads to DHF/DSS is not known. The clinical expression profiles of perforin and GrzB and the exact regulatory mechanisms that modulate the expression of perforin and GrzB during DENV infection are not clear.

MicroRNAs (miRNAs) are small non-coding RNAs of $\sim 22$ nucleotides that modulate gene expression at the post-transcriptional level by targeting the $3^{\prime}$-untranslated region $\left(3^{\prime}\right.$ UTR) of mRNA for degradation or inhibiting translation. ${ }^{16,17}$ Emerging data indicate that miRNAs participate in many physiological and pathological processes, including host-virus interactions. For example, human miR-24 modulates highly pathogenic $\mathrm{H} 5 \mathrm{~N} 1$ influenza A virus infection by targeting furin mRNA, and miR-451 directly targets the YWHAZ mRNA sequence to regulate proinflammatory cytokine responses to influenza infection. ${ }^{18,19}$ Hepatitis C virus (HCV) infection up-regulates human miR-373 expression to impair the JAK/ STAT pathway in hepatocytes, which directly targets JAK1 and IFN-regulating factor 9 (IRF9). ${ }^{20}$ A recent study demonstrated that miRNA-30e* suppressed DENV replication via promotion of NF- $\kappa \mathrm{B}$-dependent IFN production. ${ }^{21}$ However, whether and how miRNAs regulate perforin and GrzB during DENV infection is not known. The elucidation of the mechanisms of miRNA regulation of perforin and GrzB is theoretically and practically important to understand the immunopathogenesis mechanisms of DENV infection and develop novel anti-viral therapeutics.

MiR-27a*, miR-30e, and miR-378 correlate with perforin and GrzB protein expression in NK cells in cord and peripheral blood during IL-15 or IFN- $\alpha$ stimulation. ${ }^{22,23}$ The present study examined whether miR-27a*, miR-30e, and miR-378 play important roles in perforin and GrzB expression during DENV infection and further determined the roles of perforin and GrzB in DENV infection.

\section{MATERIALS AND METHODS}

\section{Human subjects}

DENV-infected patients and healthy controls (Healthy Ctrls) were recruited at the First Affiliated Hospital of Sun Yat-sen University (First Hospital of SYSU). All blood samples from DENV-infected patients or Healthy Ctrls were collected after obtaining informed written consent according to published International Health Guidelines. The Ethics Committee of First Hospital of SYSU and Zhongshan School of Medicine of SYSU approved the study protocol. DENV-infected patients experienced fever $\geqslant 5$ days, pain, subcutaneous hemorrhage, and other non-typical symptoms. Supplementary Tables S1 and S2 summarize the demographic and clinical information of dengue patients and Healthy Ctrls enrolled in this study. All sera samples were tested for DENV infection using a DENVspecific NS1-antigen ELISA Kit (Wantai, Beijing, China) and DENV serotype-specific reverse transcriptase-polymerase chain reaction (RT-PCR) (Supplementary Figure S1). Dengue-specific IgM/IgG antibodies in sera of Healthy Ctrls and patients were also detected using DENV-specific IgG and IgM capture ELISA kits (DaAn Gene, Guangzhou, China).

\section{Statistical analysis}

Statistical analyses were performed using GraphPad Prism 5.0 software (GraphPad Software, Inc, San Diego, CA, USA). Differences between groups were evaluated using a paired two-tailed Student's $t$-test or independent samples $t$-test, and $p$ values $<0.05$ were considered statistically significant. Two-tailed Pearson correlation coefficient analysis was performed using SPSS software (version 13.0, SPSS, Chicago, IL, USA).

Details for other materials and methods are shown in the "Supplementary Materials and Methods" section in Supplementary Information: Cell culture and virus; PBMCs separation and DENV infection; Antibodies; Assay for expression levels of cell-surface and intracellular molecules; NK cell isolation; Indirect immunofluorescence assay; Plaque-forming assay; Real-time quantitative PCR analysis; Synthesis and transfection of miR-378 mimic and inhibitor; Lentiviral preparation and transduction; and miR-378 agomir treatment in mice.

\section{RESULTS}

\section{DENV infection in humans induces a significant down- regulation of miR-378}

We used miRanda and TargetScan software to predict the sequence of miRNAs that potentially bind the $3^{\prime}$-UTR regions of perforin and GrzB mRNA to determine whether miRNAs regulate the expression of these human cytotoxic molecules. MiR-27a*, miR-30e, and miR-378 most potently targeted perforin and GrzB (Figure 1a), which suggests that miR-27a*, miR-30e, and miR-378 are the primary miRNAs that regulate perforin and GrzB expression. Previous studies suggested a role of miR-27a*, miR-30e, and miR-378 in the regulation of perforin and GrzB. ${ }^{22,23}$ Therefore, we chose miR-27a*, miR-30e, and miR-378 as targets to determine the relationship between miRNA expression and perforin and GrzB production.

MiRNA expression in peripheral blood mononuclear cells (PBMCs) of DENV-infected patients were evaluated using RTqPCR to determine whether miRNAs regulated perforin and GrzB expression during DENV infection. The expression of miR-27a*, miR-30e, and miR-378 was significantly downregulated in PBMCs of DENV2-infected patients (Figure 1b). Therefore, DENV infection in humans induces down-regulation of miR-27a*, miR-30e, and miR-378. Further analyses suggested that miR-27a* and miR-378 simultaneously targeted perforin and GrzB (Figure 1a), but miR-378 exhibited much higher binding potential with the $3^{\prime}$-UTR of GrzB compared to the $3^{\prime}$-UTR of perforin (Figure 1a). Therefore, we focused our efforts on understanding the relationship between miR-378 and GrzB during DENV infection. 
a

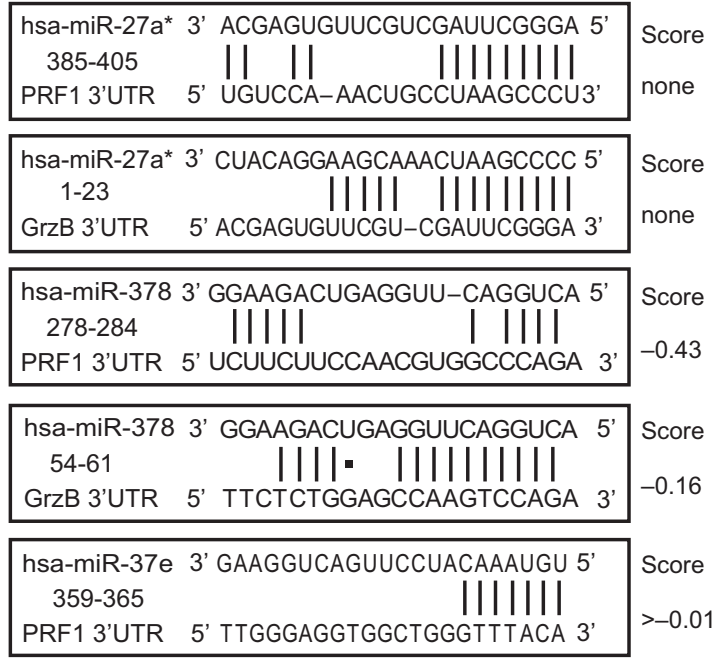

b

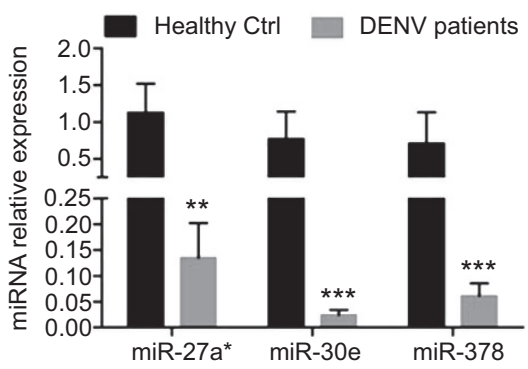

Figure 1 miR-27a*, miR-378, and miR-30e directly target perforin and/or GrzB, which are significantly down-regulated in DENV patients. (a) Human perforin and/or GrzB are putative targets of miR-27a*, miR-30e, and/or miR-378, as predicted by miRanda and TargetScan. Numbers indicate the position of nucleotides in the $3^{\prime}$-UTR that are targeted by miRNAs. (b) Pooled data show the expression levels of miR27a*, miR-30e, and miR-378 in PBMCs of DENV-infected patients (DENV patients), which is much lower than expression in PBMCs of Healthy Ctrls. Freshly isolated PBMCs were obtained from 10 DENV-infected patients and 10 Healthy Ctrls, and total RNA was extracted for analyses of miRNA expression using qPCR analysis. Data are representative of three independent experiments (mean \pm SD; independent samples $t$-test, $* * p<0.01, * * * p<0.001)$.

\section{DENV infection in humans induces an up-regulation of GrzB, and NK cells are a major source of GrzB during DENV infection}

We detected GrzB expression in DENV-infected patients using intracellular cytokine staining (ICS) and flow-cytometric analysis. Supplementary Figure S2 shows the strategy for gating $\mathrm{CD} 6^{+} \mathrm{NK}$ cells, $\mathrm{CD} 8^{+}$and $\mathrm{CD} 4^{+} \mathrm{T}$ cells. GrzB protein was significantly up-regulated in total PBMCs, $\mathrm{CD} 8^{+} \mathrm{T}$ cells, and CD56 ${ }^{+}$NK cells of DENV-infected patients, but the mRNA level of GrzB in PBMCs of DENV-infected patients was not significantly increased compared with Healthy Ctrls (Figure 2a-c; Supplementary Figure S3). These results suggest the post-transcriptional targeting of GrzB mRNA by miR-378. Consistently, a marker for the degranulation capacity of cytotoxic lymphocytes, CD107a, which is a lysozyme-associated membrane glycoprotein, ${ }^{24,25}$ was also significantly up-regulated in $\mathrm{CD}^{+} \mathrm{T}$ cells and NK cells of DENV-infected patients (Figure 2d). Given that both $\mathrm{CD}^{2} 6^{+} \mathrm{NK}$ cells and $\mathrm{CD} 8{ }^{+} \mathrm{T}$ cells produce appreciable amounts of GrzB, we therefore identified which cell sup-population was the major source of GrzB. Notably, the percentages of $\mathrm{CD}^{+} 6^{+} \mathrm{NK}\left(\mathrm{CD}^{-} \mathrm{CD}^{-} 6^{+}\right)$cells in total $\mathrm{GrzB}^{+}$PBMCs of DENV-infected patients were much higher than the percentages of $\mathrm{CD}^{+} \mathrm{CD}^{+}{ }^{-}$cells (Figure 2e), which suggests that $\mathrm{CD} 56^{+}$ NK cells are the major source of GrzB during DENV infection. Taken together, these results suggest that the down-regulation of miR-378 contributes to the up-regulation of GrzB protein expression, particularly in NK cells, but it does not influence GrzB mRNA levels in DENV-infected patients.

We determined whether the up-regulation of $\mathrm{GrzB}$ in $\mathrm{CD} 56^{+}$ NK cells was directly mediated by the down-regulation of miR378 or the secondary pathological or systemic effects during
DENV infection in humans. PBMCs were successfully infected with DENV2 in vitro (Supplementary Figure S4a-c). DENV infection induced NK and T-cell activation (Supplementary Figure S5a-c), and GrzB expression was significantly up-regulated in $\mathrm{CD}^{2} 6^{+} \mathrm{NK}$ cells, but not $\mathrm{CD} 8^{+}$or $\mathrm{CD} 4^{+} \mathrm{T}$ cells, at $24 \mathrm{~h}$ post-infection (Figure $3 \mathrm{a}-\mathrm{c}$ ). Notably, both $\mathrm{CD} 56^{\mathrm{dim}}$ and CD56 $6^{\text {bright }}$ subsets of $\mathrm{CD}^{5} 6^{+} \mathrm{NK}$ cells exhibited strong GrzB expression after DENV infection, but GrzB mRNA expression levels in isolated $\mathrm{CD} 56^{+} \mathrm{NK}$ cells were not significantly increased in the DENV-infected group (Figure 3d and e). These data suggest that miR-378 may participate in the regulation of GrzB expression at the translation level, and NK cells are a major source of GrzB expression during DENV infection.

\section{MiR-378 regulates GrzB expression in human NK cells during DENV infection}

Given that in vivo and in vitro DENV infection induced an upregulation of GrzB protein with no significant increase at the mRNA level in PBMCs, and $\mathrm{CD}^{+} 6^{+} \mathrm{NK}$ cells were the major source of GrzB, we therefore focused on $\mathrm{CD} 56^{+} \mathrm{NK}$ cells and determined whether miR-378 regulated GrzB in NK cells. qPCR analyses suggested that miR-378 and miR-30e, but not miR$27 \mathrm{a}^{*}$, were significantly down-regulated in NK cells during in vitro DENV infection (Figure $3 \mathrm{f}$ ). We performed correlation analyses of decreased miR-378 levels versus increased GrzB levels in $\mathrm{CD} 56^{\mathrm{dim}}$ or $\mathrm{CD} 56^{\text {bright }}$ subsets of NK cells with and without DENV infection to further explore whether GrzB was specifically regulated by miR-378 in NK cells. MiR-378 was negatively correlated with GrzB in NK cells during DENV infection (Figure 3g). In contrast, miR-30e did not exhibit a significant negative 
a

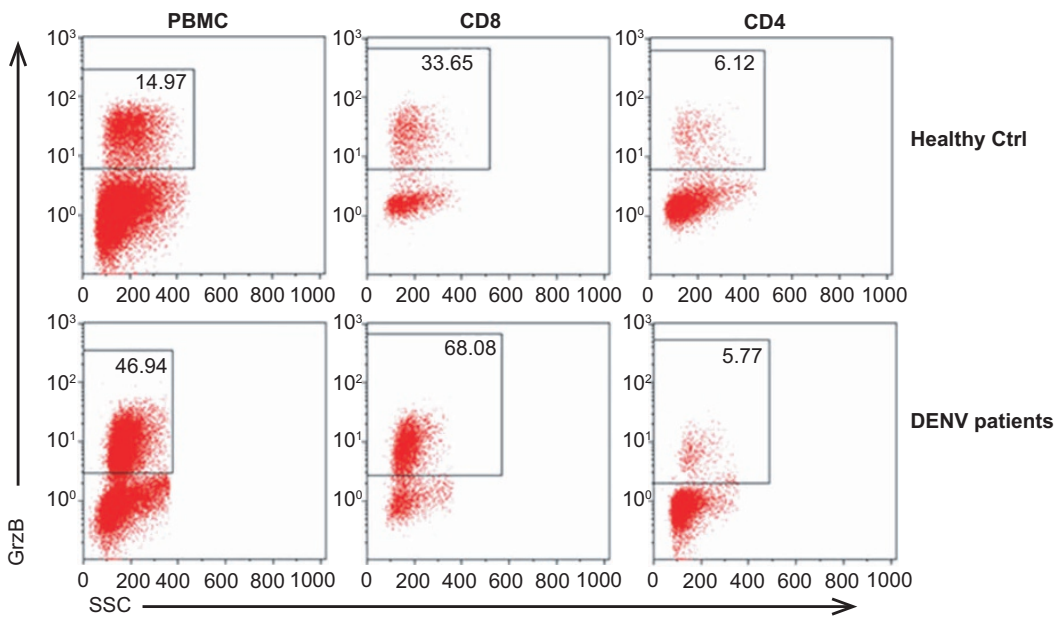

b
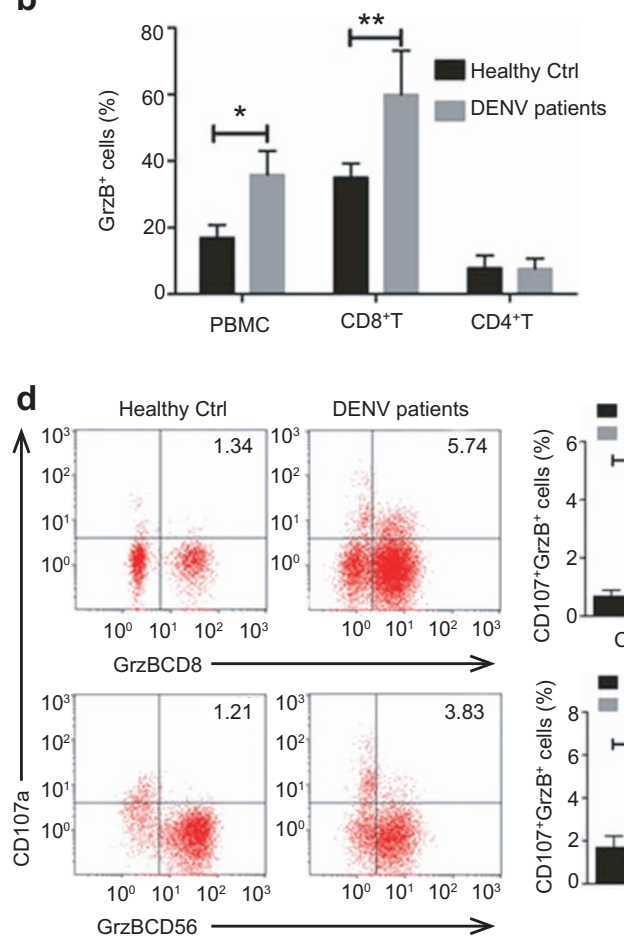

C

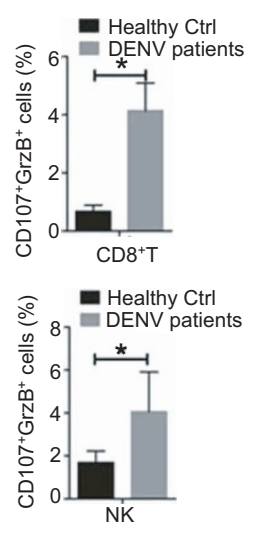

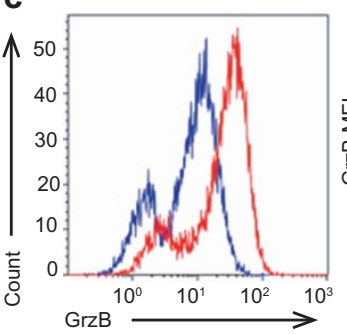

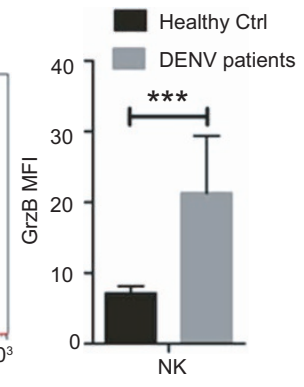

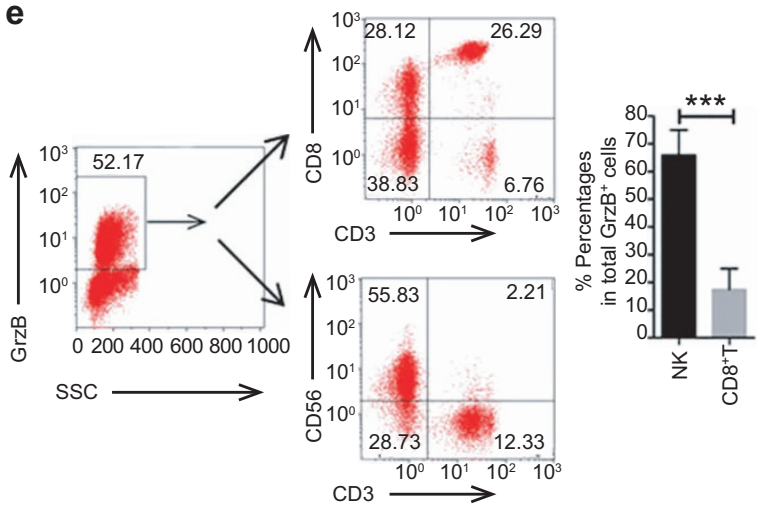

Figure 2 DENV infection induces a significant increase of GrzB expression. Freshly isolated PBMCs from 10 DENV-infected patients (DENV patients) and 10 Healthy Ctrls were directly stained with cell-surface and intracellular antibodies to analyze GrzB and CD107 expression in $\mathrm{NK}, \mathrm{CD}^{+}$and $\mathrm{CD}^{+}{ }^{+}$T cells using flow cytometry. (a) Representative flow-cytometric plots show the percentages of GrzB ${ }^{+}$cells in total PBMCs, $\mathrm{CD}^{+}$and $\mathrm{CD} 4^{+} \mathrm{T}$ cells in Healthy Ctrls and DENV patients. (b) Pooled data show that the percentages of GrzB ${ }^{+}$cells in total PBMCs and $\mathrm{CD}^{+} \mathrm{T}$ cells are significantly increased in DENV patients compared to Healthy Ctrls. (blue curve, Healthy Ctrl; red curve, DENV patients). (c) Representative flow-cytometric plots and pooled data show that the MFI of GrzB in NK $\left(\mathrm{CD}^{-} \mathrm{CD}^{-} 6^{+}\right)$cells is significantly increased in DENV patients. (d) Representative flow-cytometric plots and pooled data show that the percentages of $\mathrm{CD} 107^{+} \mathrm{GrzB}^{+}$cells in $\mathrm{CD}^{+} \mathrm{T}^{\text {or }} \mathrm{CD} 56^{+} \mathrm{NK}$ cells are significantly increased in DENV patients compared to Healthy Ctrls. (e) A representative two-tier flow-cytometric analysis and pooled data show that $\mathrm{GrzB}^{+}$cells in PBMCs of DENV patients contain more $\mathrm{CD} 3^{-} \mathrm{CD}_{5} 6^{+} \mathrm{NK}$ than $\mathrm{CD}^{+} \mathrm{CD} 8^{+}$cells. Data are representative of at least three independent experiments (mean $\pm \mathrm{SD}$; independent samples $t$-test, $* p<0.05, * * p<0.01, * * * p 0.001$ ).

correlation with GrzB in NK cells (Supplementary Figure S6). Taken together, these results suggest that miR-378 is a major regulator of GrzB expression in NK cells during DENV infection.

We determined whether miR-378 specifically regulated GrzB expression in NK cells. Primary NK cells were isolated from PBMCs of healthy donors, and the purity and sorting efficiency of isolated NK cells was $>90 \%$, as measured by flow cytometry (Figure 4a). NK cells were transfected with Cy3 fluorescein-labeled synthetic miR-378 mimics, inhibitors, control mimics, or control inhibitors. Fluorescence microscopy 
a

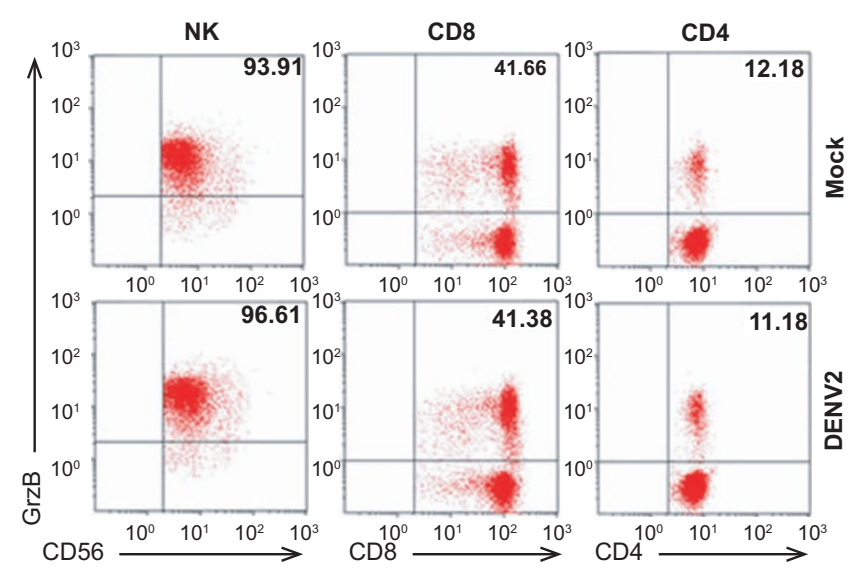

b
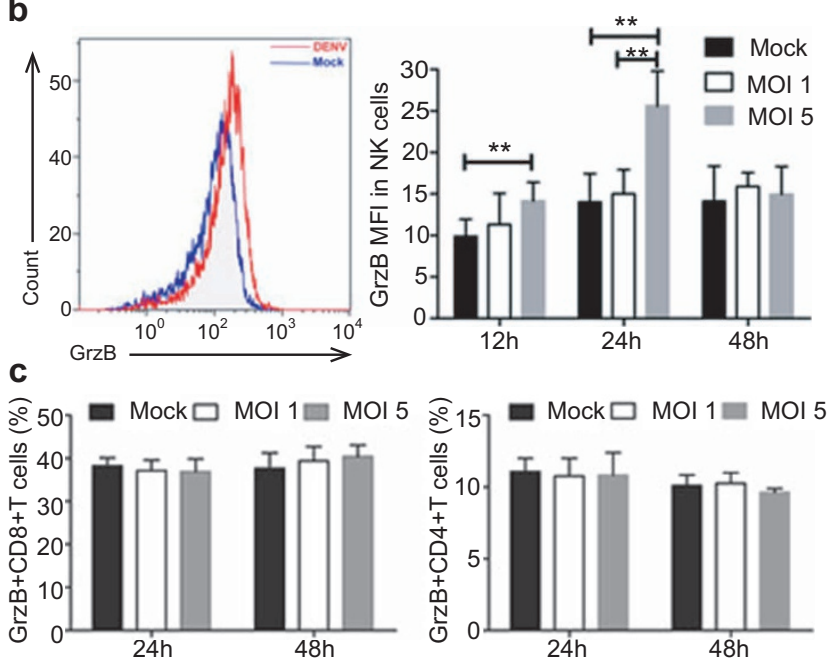
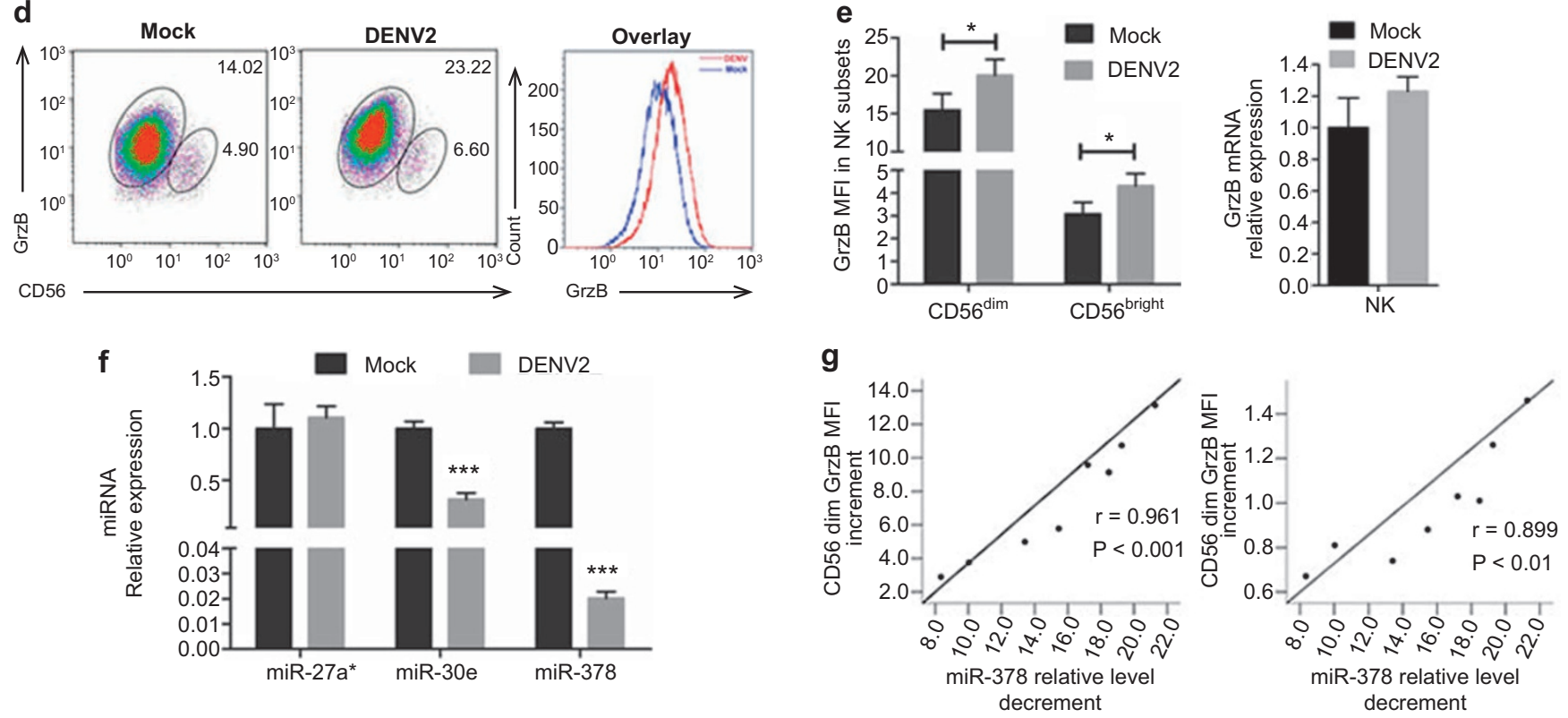

Figure 3 GrzB is negatively correlated with miR-378 in human NK cells during DENV infection. PBMCs were cultured with DENV-2 (ZSO1/01 strain) at MOIs of 0,1 , and 5 for 12,24 , and 48 hours and collected for analysis of GrzB expression in NK, CD8 ${ }^{+}$, and CD4 ${ }^{+} \mathrm{T}_{\text {cells in three groups }}$ using flow cytometry. (a) Representative flow-cytometric dot plots show the percentages of $\mathrm{GrzB}^{+}$cells in $\left.\mathrm{NK}_{(\mathrm{CD}}{ }^{-} \mathrm{CD}^{+} 6^{+}\right), \mathrm{CD}^{+}\left(\mathrm{CD} 3^{+} \mathrm{CD} 8^{+}\right) \mathrm{T}$ and $\mathrm{CD}^{+}\left(\mathrm{CD}^{+} \mathrm{CD}^{+}\right)$T cells between DENV2 - and Mock-infected cells. Numbers in each plot indicate the percentage of GrzB ${ }^{+}$cells. (b) Overlaying flow-cytometric plots of the GrzB ${ }^{+}$NK cells in DENV2 (red curve) and Mock (blue curve) groups at $24 \mathrm{~h}$ post-infection. Pooled data show that the MFI of GrzB in NK cells is significantly increased in DENV2 cells cultured within $24 \mathrm{~h}$ compared to Mock cells. (c) Pooled data show that the percentages of $\mathrm{GrzB}^{+}$cells in $\mathrm{CD}^{+}$or $\mathrm{CD}^{+}{ }^{+} \mathrm{T}$ cells are not significantly different between DENV2 and Mock cells within $48 \mathrm{~h}$ post-infection. The above representative data are from 12 samples and four independent experiments (mean $\pm \mathrm{SD}$; paired two-tailed Student's $t$-test, ** $p<0.01$ ). NK cells were also sorted from DENV-infected PBMCs at an MOI of 5 for $24 \mathrm{~h}$ as a non-infected control group. (d) Represented flow-cytometric plots show GrzB expression levels in sorted NK $\left(\mathrm{CD}^{-} \mathrm{CD}^{+} 6^{+}\right)$cells between DENV2 and Mock groups, and numbers indicate the MFI of GrzB in $\mathrm{CD56}^{\text {dim }}$ and CD56 $6^{\text {bright }}$ subsets in each plot. The overlaying plot displays GrzB ${ }^{+}$cells in CD56 ${ }^{\text {dim }}$ subsets in DENV2 (red curve) and Mock (blue curve) groups. (e) Pooled data show that GrzB expression levels in CD56 ${ }^{\text {dim }}$ or CD56 bright subsets are significantly increased in DENV2 (left), but the mRNA levels in isolated NK cells between the two groups were not significantly different (right). (f) Pooled data of qPCR analysis show that in vitro DENV infection induces a down-regulation of miR-30 and miR-378, but not miR-27a*, in NK cells. (g) GrzB is negatively correlated with miR-378 in $\mathrm{CD}^{\mathrm{dim}}$ or CD56 ${ }^{\text {bright }} \mathrm{NK}$ cells during in vitro DENV infection. The decrement values of relative expression of miR-378 versus GrzB using mean fluorescence intensity (MFI) increment values were conducted for correlational statistical analyses using the Pearson correlation coefficient analysis to determine the correlation between miR-378 and GrzB expression. Decrement values of miR-378 were determined by subtracting miR-378 relative expression values in DENV2 cells from the expression values in Mock cells. Increased values of GrzB MFI were determined by subtracting GrzB MFI values in Mock cells from the values in DENV2 cells. The left panel shows the correlation between GrzB and miR-378 in CD56 $^{\text {dim }}$ NK cells $(p<0.001)$, and the right panel shows the correlation between GrzB and miR-378 in CD56 ${ }^{\text {bright }} \mathrm{NK}$ cells $(p<0.01)$. The above representative data are from eight samples and three independent experiments (mean $\pm \mathrm{SD}$; paired two-tailed Student's $t$-test, $* p<0.05, * * * p<$ 0.001). DENV2, DENV-infected group (MOI 5); Mock, non-infected group. 
a

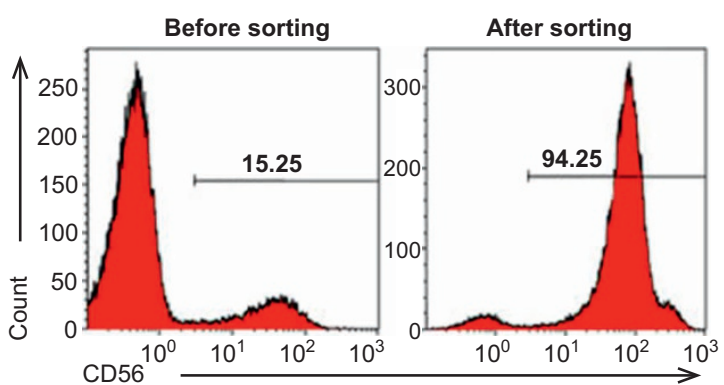

d 든

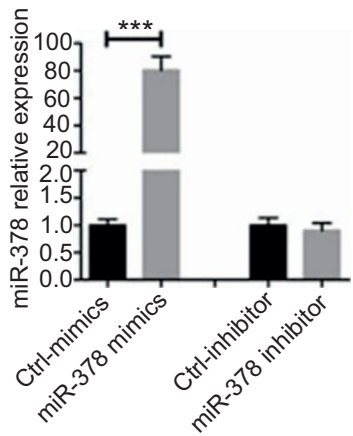

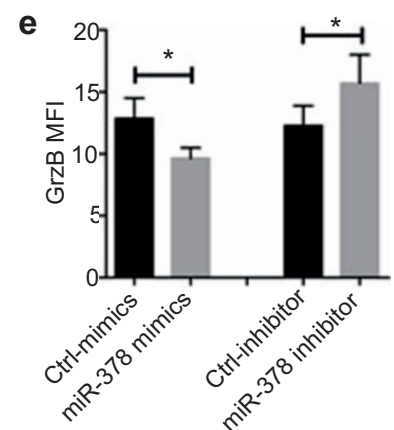

b

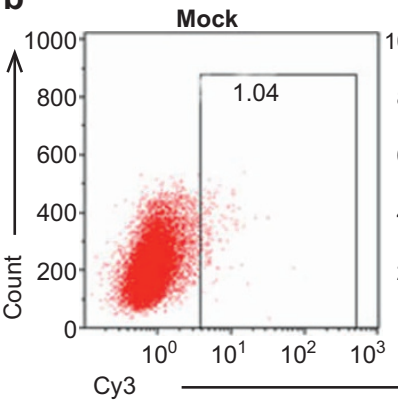

c
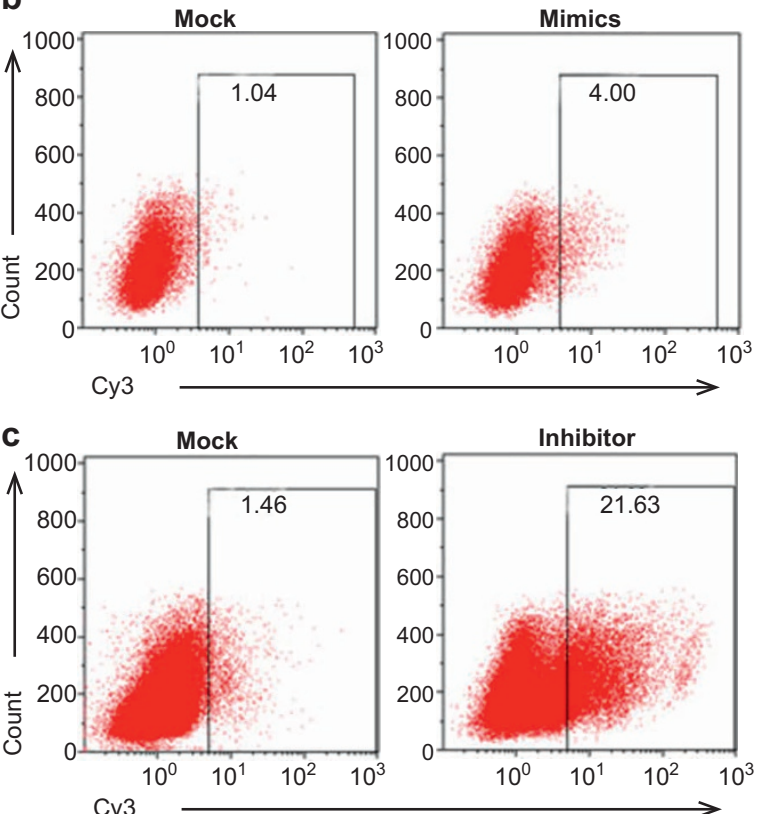

f
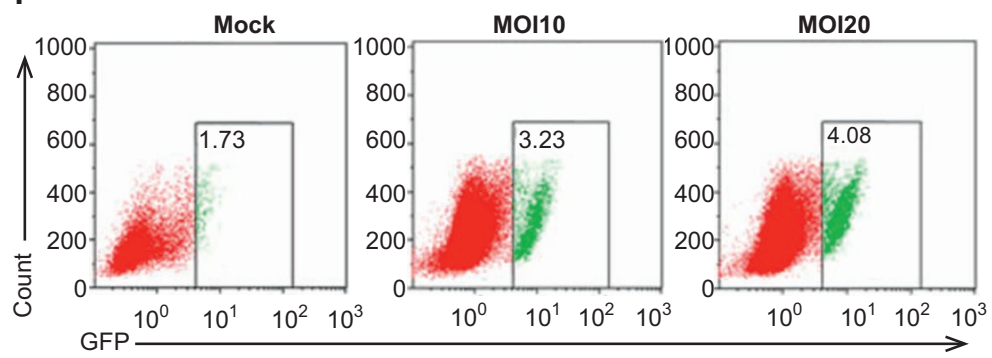

g

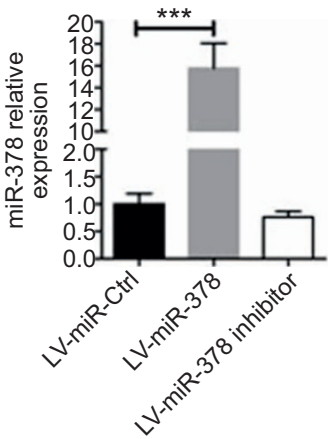

h

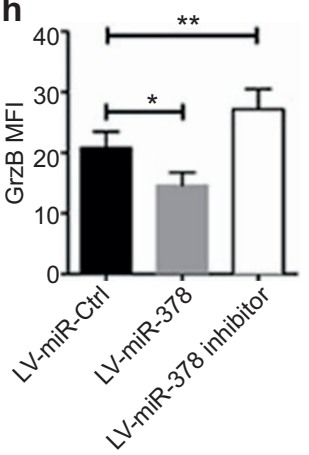

Figure 4 GrzB is negatively regulated by miR-378 in human primary NK cells. Freshly sorted primary NK cells from PBMCs of healthy controls were transfected with Cy3 fluorescein-labeled miR-378 mimics ( $50 \mathrm{nM}$ ), control mimics ( $50 \mathrm{nM}$ ), miR-378 inhibitors ( $100 \mathrm{nM}$ ), or control inhibitors (100 $\mathrm{nM}$ ). (a) Representative flow-cytometric plots show the purity of sorted NK cells. (b and $\mathbf{c}$ ) Representative flow-cytometric plots show the transfection efficiency of miR-378 mimics and inhibitors. Numbers indicate the percentages of positive cells in each plot. (d and e) The pooled data show the levels of miR-378 (left) and GrzB protein (right) determined using RT-qPCR and flow-cytometric assays in NK cells transfected with miR-378 mimics or miR-378 inhibitors compared with transfection with Control mimics or Control inhibitors. The sorted NK cells were transduced using lentivirus with vectors of hsa-miR-378 (LV-miR-378), hsa-miR-378 inhibitor (LV-miR-378 inhibitor), or LV-miR-negative control (LV-miRCtrl) for seven days. Cells were collected for analyses of miR-378 and GrzB levels using RT-qPCR and flow cytometry, respectively. (f) Representative flow-cytometric plots show the rates of GFP ${ }^{+}$NK cells after transduction of LV-miR-Ctrl at MOls of 0, 10, and 20. (g) The pooled data show that miR-378 expression is significantly increased in NK cells transduced with LV-miR-378. (h) The MFI of GrzB is down-regulated in NK cells transduced with LV-miR-378, but it is up-regulated in NK cells transduced with the LV-miR-378 inhibitor compared to LV-miR-Ctrl. Representative data are at least three independent experiments with one donor in every experiment (mean \pm SD; paired two-tailed Student's t-test, $* p<0.05, * * p<0.01, * * * p<0.001)$. Mock, non-transfected or non-transduced group.

and flow-cytometric analyses confirmed that miR-378 mimics and inhibitors were transfected in NK cells (Figure $4 \mathrm{~b}$ and $\mathrm{c}$ ). qPCR analysis revealed that miR-378 expression was significantly increased in NK cells after transfection of miR-378 mimics compared with control mimics (Figure 4d). ICS/ flow-cytometric analyses of GrzB showed that transfection of miR-378 mimics induced a significant decrease in the MFI of GrzB in NK cells compared with control mimics (Figure 4e). Conversely, transfection of miR-378 inhibitors induced a sig- nificant increase in the MFI of GrzB in NK cells compared to control inhibitors (Figure 4e). Furthermore, we used a lentiviral transduction method to stably overexpress miR-378 or inhibit intrinsic miR-378 in human primary $\mathrm{NK}$ cells (Figure $4 \mathrm{f}$ and $\mathrm{g}$ ). Similar decreases in the MFI of GrzB after transduction of a lentiviral vector overexpressing miR-378 and increases in the MFI of GrzB in NK cells after transduction of a lentiviral vector that inhibited intrinsic miR-378 compared to controls were observed (Figure $4 \mathrm{~h}$ ). These results collectively 
suggest that miR-378 regulates GrzB expression in human NK cells during DENV infection.

Treatment of mice with an miR-378 agomir suppresses GrzB expression and facilitates DENV replication

We observed that DENV infection induced an up-regulation of GrzB, but whether GrzB played a protective or immunopathological role during in vivo DENV infection was not clear. MiR378 was a major regulator of GrzB expression in our model. Therefore, we examined whether the overexpression of miR378 down-regulated GrzB expression and further induced a protective or immunopathological effect in DENV-infected mice. Bioinformatics analyses suggested that miR-378 targeted the $3^{\prime}$ UTR region of mouse GrzB mRNA (Figure 5a). Furthermore, treatment of mice with an miR-378 agomir, which induces overexpression of miR-378 in mouse spleens and peripheral blood (Figure 5b), significantly reduced the percentages of $\mathrm{GrzB}^{+} \mathrm{NK}$ cells in spleens compared to treatment with a control agomir
(Figure $5 \mathrm{c}$ and $\mathrm{d}$ ). These results suggested that the overexpression of miR-378 inhibited GrzB expression in DENV-infected mice. Notably, the down-regulation of GrzB was associated with a significant up-regulation of viral RNA levels and $M$ and $E$ gene expression of DENV2 in spleens and brains (Figure 5e and f). The reduced expression of GrzB following miR-378 treatment was associated with increased expression of DENV $M$ and $E$ transcripts, which suggests that GrzB contributes to the control of DENV replication in vivo.

\section{Discussion}

GrzB plays an important role in the control of viral infection, but the regulatory mechanisms involved in the release of GrzB and the exact roles of GrzB in DENV infection are not clear. Accumulating evidence suggests that miRNAs are important participants in host-virus immune regulation. ${ }^{26-33}$ For example, recent studies suggested that miR-122 facilitated HCV replication in livers, and miR-323, miR491, miR-654 a

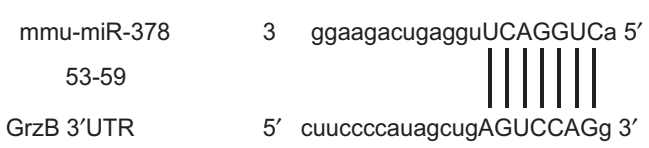

C

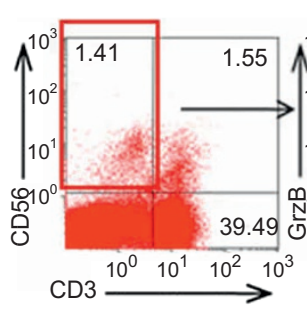

PBS

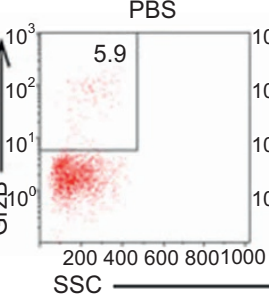

Ctrl-agomir

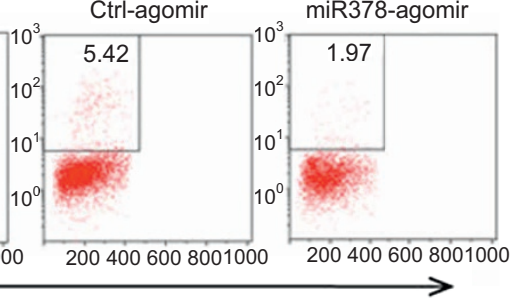

e

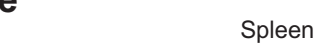

b

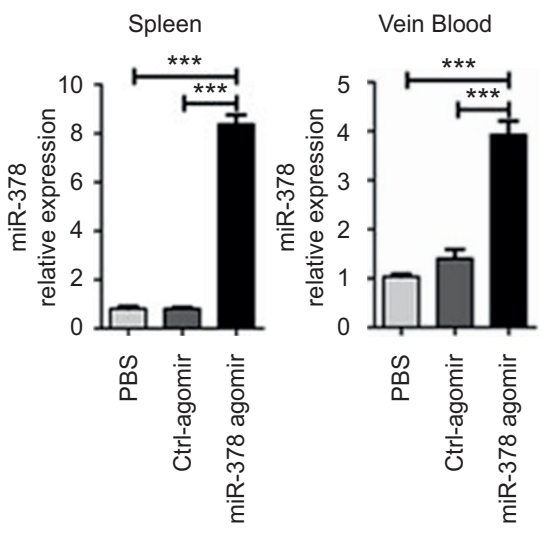

d

Spleen

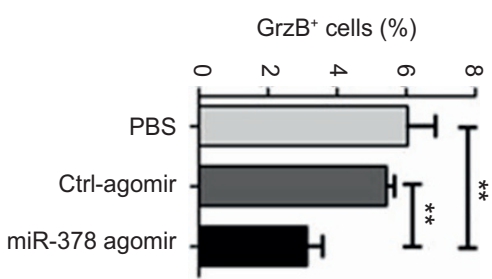

PBS

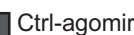

miR-378 agomir

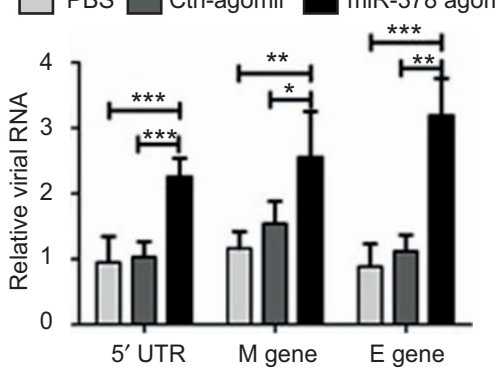

f

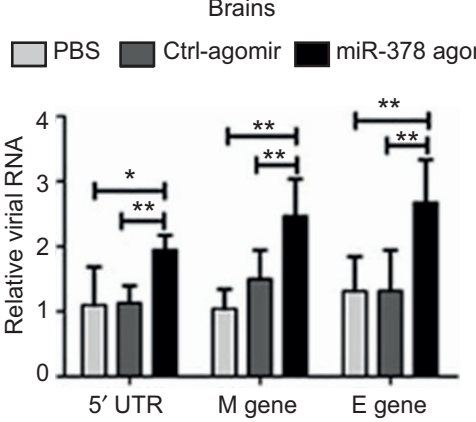

Figure 5 miR-378 overexpression in mice using an miR-378 agomir inhibits GrzB expression and promotes DENV replication. Mice were injected intraperitoneally with an miR-378 agomir ( $5 \mathrm{ng}$ ), Ctrl-agomir ( $5 \mathrm{ng}$ ) or an equal volume of PBS on days 0,3 , and 6 . Mice were inoculated with the DENV-2 ZS01/01 strain ( $10^{6} \mathrm{pfu} / \mathrm{mouse}$ ) after the third treatment. Brains, spleens, and peripheral blood of mice were obtained for analyses of miR378 expression and viral RNA levels at $24 \mathrm{~h}$ post-infection using qPCR. (a) Prediction of the binding sites between the $m m u$-miR-378 seed sequence and mmu-GrzB mRNA sequence by miRBase and TargetScanMouse. Numbers indicate the position of nucleotides of mouse GrzB mRNA 3'-UTR that are targeted by miR-378. (b) The pooled data show that miR-378 expression in spleens and peripheral blood in mice treated with miR-378 agomir are significantly higher than mice treated with the Ctrl-agomir or PBS. (c) Represented flow-cytometric plots show the percentages of $\mathrm{GrzB}^{+}$of $\mathrm{NK}_{(\mathrm{CD}}{ }^{-} \mathrm{CD} 6^{+}$) cells in spleens of mice treated with the miR-378 agomir, Ctrl-agomir or PBS. (d) Pooled data show that the percentages of $\mathrm{GrzB}^{+}$of $\mathrm{NK}$ cells in spleens are significantly decreased in mice treated with the miR-378 agomir compared to mice treated with Ctrl-agomir or PBS. DENV2 RNA levels in spleens (e) and brains (f) of mice treated with miR-378 agomir via detection of viral $M$ and $E$ genes and the 5 '-UTR sequence are significantly higher than mice treated with Ctrl-agomir or PBS. Representative data are at least three independent experiments with four to six mice per group (mean $\pm \mathrm{SD}$; independent samples $t$-test, ${ }^{*} p<0.05,{ }^{*} p<0.01,{ }^{* * *} p<0.001$ ). 
inhibited $\mathrm{H} 1 \mathrm{~N} 1$ influenza A virus replication via binding to the PB1 gene. ${ }^{34,35}$ However, whether and how some miRNAs play roles in the regulation of GrzB expression in DENV-infected patients is not clear. Whether these cytotoxic molecules, including GrzB and perforin, play a protective role in facilitating the control of DENV replication or a detrimental role by contributing to the immunopathogenesis of severe DENV infection, such as DHF/DSS, is also not clear.

The present study found that miR-378 expression was significantly down-regulated in PBMCs derived from DENV-infected patients and DENV-infected human NK cells in vitro. We further demonstrated that DENV infection induced an up-regulation of GrzB, and miR-378 was a major regulator of GrzB expression in NK cells. The development of an animal model that mimics human dengue immunopathogenesis remains technically challenging, but our in vivo study in DENV-infected mice suggested that the overexpression of miR-378, which significantly inhibited GrzB expression, was associated with the enhanced replication of DENV in multiple organs, including spleens and brains, which implicates a protective potential of GrzB against DENV infection. We found that treatment with miR-378 agomir depressed GrzB expression in DENV-infected mice, and depressed expression GrzB was associated with an increased expression of DENV $M$ and $E$ transcripts. Therefore, we cannot exclude the possibility that other immune factors act synergistically with GrzB to facilitate the control of DENV replication in mice. This synergism is possible because miR-378 may target multiple mRNAs, ${ }^{36-38}$ and other studies are necessary to confirm our findings of an association of enhanced DENV $M$ and $E$ gene replications and depressed GrzB expression. GrzB may also contribute to the control of DENV replication through a secondary effect by triggering or regulating the expression of other immune factors that are critical for the control of DENV replication. Therefore, future in-depth studies are required to further understand the roles of GrzB in DENV viral replication.

Previous studies suggested that miR-378 is involved in the regulation of immune function and the cytotoxicity of NK cells via regulation of GrzB expression at translation levels. ${ }^{23}$ However, the exact roles of miR-378 in host-DENV interactions are not known. The present study provides an important line of evidence to help explain the possible mechanisms of host miRNA participation in the modulation of DENV-triggered immune responses.

We found that DENV infection in humans induced a downregulation of miR-378, but the processes that result in this down-regulation were not elucidated. Previous studies on Type I IFN signaling offer possibilities. Recent studies suggested that Type I IFN induced by DENV infection ${ }^{39}$ further induces $\mathrm{GrzB}$ and perforin expression in murine NK cells, which may be required for NK cell activation in response to viral infection. ${ }^{40,41}$ IFN- $\alpha$-activated human NK cells may enhance GrzB expression via a down-regulation of miR-378 expression. ${ }^{23}$ Therefore, the down-regulation of miR-378 in DENV-infected patients may be a product of DENV-induced Type I IFN signaling, but this hypothesis requires further investigation. However, other possible regulatory factors may affect miR-378 expression, such as systemic or secondary effects of DENV infection, particularly in severe DENV infection (DHF/DSS), which is characterized by large-scale hemorrhage, high fever and multiple organ failure. Further studies are required to investigate the exact mechanisms underlying alternations in host miRNA expression induced by DENV infection.

Notably, miR-378, as a post-transcriptional regulator, is not merely confined to the processes of NK cell regulation; it may also play diverse roles in regulating the activation of other cytotoxic cellular subsets, such as $\mathrm{CD} 8^{+} \mathrm{T}$ cells. ${ }^{23} \mathrm{~A}$ recent study suggested that the up-regulation of GrzB expression mediated by Type I IFN signaling was required for the increased cytolytic activity of virus-specific $\mathrm{CD} 8^{+} \mathrm{T}$ cells. ${ }^{42}$ We cannot exclude the possibility that miR-378 also participates in the regulation of GrzB expression in $\mathrm{CD}^{+}{ }^{+} \mathrm{T}$ cells during DENV infection in a Type I IFN signaling-dependent manner.

A previous study demonstrated that CD107a molecule expression was required for ex vivo antigenic re-stimulation, ${ }^{43}$ but we detected significant $\mathrm{CD} 107 \mathrm{a}^{+}$cells in PBMCs derived from dengue patients without additional ex vivo antigenic restimulation. This difference likely occurred because acute dengue viral infection in vivo may have provided adequate stimulation, and an ex vivo antigenic re-stimulation for the expression of CD107a was rendered unnecessary.

Taken together, the current study demonstrates the involvement of miR-378 in the regulation of immune responses of GrzB production by human NK cells during acute DENV infection. This work extends our understanding of the roles of miR378 in interactions between hosts and DENV and provides a biological basis for the antiviral activities of GrzB, which are important to improving our knowledge of dengue immunopathogenesis and developing potential antiviral therapeutics.

\section{ACKNOWLEDGEMENTS}

We thank Xiaobo Li for technical assistance of flow-cytometric analyses. This study was supported by Guangzhou Municipality Commission for Science and Technology Innovation Grant 2015080200263, the Joint National Nature Science Foundation of China and Guangdong Science Foundation Program (U1132002 and U0632002), Natural Science Foundation of Guangdong Province (2013A020229004), and National Nature Science Foundation of China (31270974 and 30972763).

Supplementary Information of this article can be found on the Cellular \& Molecular Immunology's website: http://www.nature.com/cmi.

1 Deen JL, Harris E, Wills B, Balmaseda A, Hammond SN, Rocha C et al. The WHO dengue classification and case definitions: time for a reassessment. Lancet 2006; 368: 170-173.

2 Halstead SB, Nimmannitya S, Cohen SN. Observations related to pathogenesis of dengue hemorrhagic fever. IV. Relation of disease severity to antibody response and virus recovered. Yale J Biol Med 1970; 42: 311-328.

3 Guzman MG, Halstead SB, Artsob H, Buchy P, Farrar J, Gubler DJ et al. Dengue: a continuing global threat. Nat Rev Microbiol 2010; 8: S7-S16. 
4 Espina LM, Valero NJ, Hernandez JM, Mosquera JA. Increased apoptosis and expression of tumor necrosis factor-alpha caused by infection of cultured human monocytes with dengue virus. Am J Trop Med Hyg 2003; 68: 48-53.

5 Villar LA, Gelvez RM, Rodriguez JA, Salgado D, Parra B, Osorio L et al. [Biomarkers for the prognosis of severe dengue]. Biomedica 2013; 33: 108-116.

6 Prestwood TR, Morar MM, Zellweger RM, Miller R, May MM, Yauch LE et al. Gamma interferon (IFN-gamma) receptor restricts systemic dengue virus replication and prevents paralysis in IFN-alpha/beta receptor-deficient mice. J Virol 2012; 86: 12561-12570.

7 Mathew A, Rothman AL. Understanding the contribution of cellular immunity to dengue disease pathogenesis. Immunol Rev 2008; 225: 300-313.

8 Mongkolsapaya J, Duangchinda T, Dejnirattisai W, Vasanawathana S, Avirutnan $\mathrm{P}$, Jairungsri A et al. T cell responses in dengue hemorrhagic fever: are cross-reactive T cells suboptimal? J Immunol 2006; 176: 3821-3829.

9 Voskoboinik I, Smyth MJ, Trapani JA. Perforin-mediated target-cell death and immune homeostasis. Nat Rev Immuno/2006; 6: 940-952.

10 van de Berg PJ, van Leeuwen EM, ten Berge IJ, van Lier R. Cytotoxic human CD4(+) T cells. Curr Opin Immunol 2008; 20: 339-343.

11 Azeredo EL, De Oliveira-Pinto LM, Zagne SM, Cerqueira DI, Nogueira RM, Kubelka CF. NK cells, displaying early activation, cytotoxicity and adhesion molecules, are associated with mild dengue disease. Clin Exp Immunol 2006; 143: 345-356.

12 Petitdemange C, Wauquier N, Rey J, Hervier B, Leroy E, Vieillard V. Control of acute dengue virus infection by natural killer cells. Front Immunol 2014; 5: 209.

13 Weiskopf D, Angelo MA, de Azeredo EL, Sidney J, Greenbaum JA, Fernando AN et al. Comprehensive analysis of dengue virus-specific responses supports an HLA-linked protective role for CD8+ T cells. Proc Natl Acad Sci U S A 2013; 110: E2046-E2053.

14 Yauch LE, Zellweger RM, Kotturi MF, Qutubuddin A, Sidney J, Peters $\mathrm{B}$ et al. A protective role for dengue virus-specific CD8+ T cells. $\mathrm{J}$ Immunol 2009; 182: 4865-4873.

15 Torrentes-Carvalho A, Marinho CF, de Oliveira-Pinto LM, de Oliveira DB, Damasco PV, Cunha RV et al. Regulation of T lymphocyte apoptotic markers is associated to cell activation during the acute phase of dengue. Immunobiology 2014; 219: 329-340.

16 Bartel DP. MicroRNAs: target recognition and regulatory functions. Cell 2009; 136: 215-233.

17 Ambros V. The functions of animal microRNAs. Nature 2004; 431: 350-355.

18 Rosenberger CM, Podyminogin RL, Navarro G, Zhao GW, Askovich PS, Weiss MJ et al. miR-451 regulates dendritic cell cytokine responses to influenza infection. J Immunol 2012; 189: 5965-5975.

19 Loveday EK, Diederich S, Pasick J, Jean F. Human microRNA-24 modulates highly pathogenic avian-origin $\mathrm{H} 5 \mathrm{~N} 1$ influenza $A$ virus infection in A549 cells by targeting secretory pathway furin. J Gen Virol 2015; 96: 30-39.

20 Mukherjee A, Di Bisceglie AM, Ray RB. Hepatitis C virus mediated enhancement of miR-373 impairs JAK/STAT signaling pathway. J Virol 2015; 89: 3356-3365.

21 Zhu X, He Z, Hu Y, Wen W, Lin C, Yu J et al. MicroRNA-30e* suppresses dengue virus replication by promoting NF-kappaBdependent IFN production. PLoS Negl Trop Dis 2014; 8: e3088.

22 Kim TD, Lee SU, Yun S, Sun HN, Lee SH, Kim JW et al. Human microRNA-27a* targets Prf1 and GzmB expression to regulate NKcell cytotoxicity. Blood 2011; 118: 5476-5486.

23 Wang $P$, Gu Y, Zhang Q, Han Y, Hou J, Lin L et al. Identification of resting and type I IFN-activated human NK cell miRNomes reveals microRNA-378 and microRNA-30e as negative regulators of NK cell cytotoxicity. J Immunol 2012; 189: 211-221.

24 Betts MR, Brenchley JM, Price DA, De Rosa SC, Douek DC, Roederer $M$ et al. Sensitive and viable identification of antigen-specific CD8+ T cells by a flow cytometric assay for degranulation. J Immunol Methods 2003; 281: 65-78.

25 Betts MR, Koup RA. Detection of T-cell degranulation: CD107a and b. Methods Cell Biol 2004; 75: 497-512.
26 Chiang K, Sung TL, Rice AP. Regulation of cyclin T1 and HIV-1 Replication by microRNAs in resting CD4 $+\mathrm{T}$ lymphocytes. $J$ Virol 2012; 86: 3244-3252.

27 Cullen BR. MicroRNAs as mediators of viral evasion of the immune system. Nat Immunol 2013; 14: 205-210.

28 Heiss BL, Maximova OA, Thach DC, Speicher JM, Pletnev AG. MicroRNA targeting of neurotropic flavivirus: effective control of virus escape and reversion to neurovirulent phenotype. J Virol 2012; 86: 5647-5659.

29 Li Y, Masaki T, Lemon SM. miR-122 and the Hepatitis C RNA genome: more than just stability. RNA Biol 2013; 10: 919-923.

30 Li Y, Masaki T, Yamane D, McGivern DR, Lemon SM. Competing and noncompeting activities of miR-122 and the $5^{\prime}$ exonuclease Xrn1 in regulation of hepatitis $C$ virus replication. Proc Natl Acad Sci U S A 2013; 110: 1881-1886.

31 Terrier O, Textoris J, Carron C, Marcel V, Bourdon JC, Rosa-Calatrava M. Host microRNA molecular signatures associated with human H1N1 and H3N2 influenza A viruses reveal an unanticipated antiviral activity for miR-146a. J Gen Virol 2013; 94: 985-995.

32 Smith JL, Grey FE, Uhrlaub JL, Nikolich-Zugich J, Hirsch AJ. Induction of the cellular microRNA, Hs 154, by West Nile virus contributes to virus-mediated apoptosis through repression of antiapoptotic factors. J Virol 2012; 86: 5278-5287.

33 Wu S, He L, Li Y, Wang T, Feng L, Jiang L et al. miR-146a facilitates replication of dengue virus by dampening interferon induction by targeting TRAF6. J Infect 2013; 67: 329-341.

34 Jopling CL, Yi M, Lancaster AM, Lemon SM, Sarnow P. Modulation of hepatitis $C$ virus RNA abundance by a liver-specific MicroRNA. Science 2005; 309: 1577-1581.

35 Song L, Liu H, Gao S, Jiang W, Huang W. Cellular microRNAs inhibit replication of the H1N1 influenza A virus in infected cells. J Virol 2010; 84: 8849-8860.

36 Pan D, Mao C, Quattrochi B, Friedline RH, Zhu LJ, Jung DY et al. MicroRNA-378 controls classical brown fat expansion to counteract obesity. Nat Commun 2014; 5: 4725.

37 Pan B, Toms D, Shen W, Li J. MicroRNA-378 regulates oocyte maturation via the suppression of aromatase in porcine cumulus cells. Am J Physiol Endocrinol Metab 2015; 308: E525-E534.

38 Yu BL, Peng XH, Zhao FP, Liu X, Lu J, Wang L et al. MicroRNA-378 functions as an onco-miR in nasopharyngeal carcinoma by repressing TOB2 expression. Int J Oncol 2014; 44: 1215-1222.

39 Simmons CP, Popper S, Dolocek C, Chau TN, Griffiths M, Dung NT et al. Patterns of host genome-wide gene transcript abundance in the peripheral blood of patients with acute dengue hemorrhagic fever. $J$ Infect Dis 2007; 195: 1097-1107.

40 Biron CA, Nguyen KB, Pien GC, Cousens LP, Salazar-Mather TP. Natural killer cells in antiviral defense: function and regulation by innate cytokines. Annu Rev Immunol 1999; 17: 189-220.

41 Martinez J, Huang X, Yang Y. Direct action of type I IFN on NK cells is required for their activation in response to vaccinia viral infection in vivo. J Immunol 2008; 180: 1592-1597.

42 Kohlmeier JE, Cookenham T, Roberts AD, Miller SC, Woodland DL. Type I interferons regulate cytolytic activity of memory CD8(+) T cells in the lung airways during respiratory virus challenge. Immunity 2010; 33: 96-105.

43 Makedonas G, Hutnick N, Haney D, Amick AC, Gardner J, Cosma G et al. Perforin and IL-2 upregulation define qualitative differences among highly functional virus-specific human CD8 T cells. PLoS Pathog 2010; 6: e1000798.

This work is licensed under a Creative Commons Attribution-NonCommercial-ShareAlike 3.0 Unported License. The images or other third party material in this article are included in the article's Creative Commons license, unless indicated otherwise in the credit line; if the material is not included under the Creative Commons license, users will need to obtain permission from the license holder to reproduce the material. To view a copy of this license, visit http://creativecommons.org/licenses/by-nc-sa/3.0/ 